\title{
OFDM Channel Estimation Algorithms based on Discrete Fourier Transform
}

\author{
Li Wenjuan $^{1}$ and Zhao Ruiyu ${ }^{1}$ \\ ${ }^{1}$ College of Mobile Telecommunications Chongqing University of Posts and \\ Telecom, Hechuan, Chongqing, China. \\ E-mail: 38061855@qq.com
}

\begin{abstract}
In allusion to such problem as unsatisfactory accuracy of the traditional laser communication LTE channel estimation algorithm, an LTE-OFDM multi-layer channel estimation algorithm based on least square $2 D$ spread spectrum is proposed in this paper. Firstly, $2 D$ spread spectrum estimation is adopted to design the weighted $2 D$ spread spectrum estimation algorithm framework under multi-layer time-frequency domain LTE-OFDM resource model; secondly, in order to improve the accuracy of $2 D$ spread spectrum estimation, the linear least square algorithm is adopted for weight parameter estimation and $2 D$ spread spectrum power normalization; finally, through the comparison in the simulation calculation example, it is indicated that the proposed algorithm is superior to the standard $2 D$ spread spectrum algorithm and the linear least square algorithm in the aspect of channel estimation effect.
\end{abstract}

Keywords: Accuracy; Spread spectrum; Channel estimation; Communication; Resource model

\section{Introduction}

In the traditional algorithm researches, the accurate and reliable channel estimation is always the key point for solving the problem regarding massive data throughout. The traditional channel state information (CSI) can be obtained through comparing the training data sequences of the transmitting terminal and the receiving terminal. In order to conquer the fluctuation of the channel transfer function in time-frequency domain, the training data sequences shall be usually twodimensional sequences ${ }^{[1-4]}$. Therefore, the training sequences or the common pilotfrequency code elements always occupy certain time and frequency, but they cannot carry any effective load data resources. Moreover, for the pilot-frequency code elements existing in the time-frequency resources, the unknown CSI value thereof can be only traditionally estimated through interpolation. Obviously, the network model with more pilot-frequency code elements and denser time-frequency estimation can be adopted to obtain more reliable CSI value, but the complexity of the model and the calculation is correspondingly increased, thus unfavorable for satisfying the real-time requirement for signal transmission. Even so, the effect is still unsatisfactory, and the high network calculation overhead is inconsistent with the low network throughput ${ }^{[5]}$. Therefore, the scholars in this industry have carried out deep researches. For example, the orthogonal frequency division multiplexing (OFDM) system is adopted in literatures [6 7] on the basis of the two-dimensional signal thought; compared with three-partition frequency reuse, the single-partition frequency reuse can significantly improve the communication network throughput. However, the single-partition reuse cannot obtain any additional frequency band benefit and can excessively increase the bandwidth. Therefore, in allusion to such characteristics as time-frequency domain anti-interference robustness of $2 \mathrm{D}$ spread 
spectrum estimation as well as the parameter selection flexibility and low calculation complexity of the linear LS estimation, this paper mainly aims at proposing the laser communication LTE-OFDM multi-layer channel estimation algorithm based on linear least square two-dimensional spread spectrum.

\section{System Model}

In order to introduce the principle of the propose method, it is considered to implement the algorithm in LTE physical layer system model. This section mainly aims at describing the characteristic relation between the laser communication LTE model and the proposed method. The frequency bandwidth (BW, $B W \in[1.4,3,5,10,15,20]$ ) is adopted for LTE, and the bandwidth (BW) is related to the quantity of the corresponding resource blocks ( $N_{r b} \in[6,15,25,50,75,100]$ ), wherein the so called resource block is composed of 12 subcarriers with the interval of $15 \mathrm{kHz}$. Specifically, the system model is as shown in Figures 1 (a) and (b).

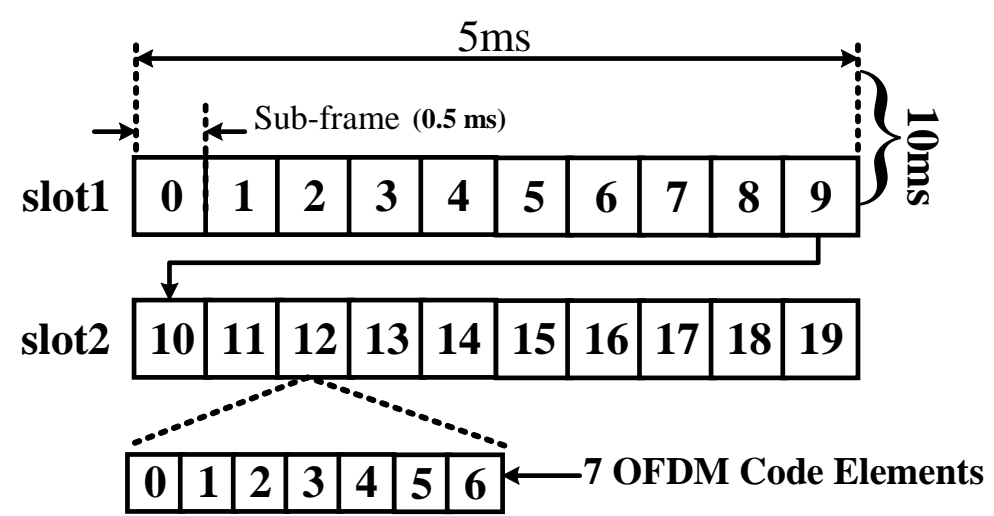

(a) Time domain LTE resource description

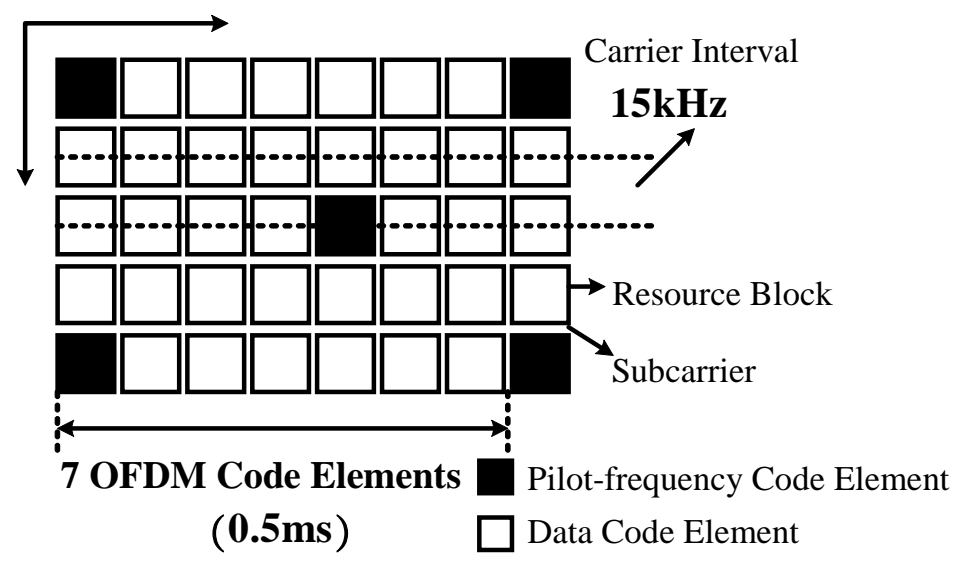

(b) Time-frequency domain LTE resource description

\section{Figure 1. LTE System Model Description}

As shown in Figure 1 (a), the resource is described as frame form in the time domain, wherein each frame lasts for $10 \mathrm{~ms}$ and can be divided into two slots, and each slot further includes 10 sub-frames with equal duration as $0.5 \mathrm{~ms}$. Specifically, a sub-frame is composed of 7 OFDM (Orthogonal Frequency Division Multiplexing) code elements, and a resource block is composed of one OFDM code element and one subcarrier. As shown in Figure 1 (b), LTE time-frequency grid 
includes pilot-frequency code elements which occupy certain resources. These pilot-frequency code elements are arranged according to relevant standards, but any particular channel estimation technology is not required by LTE, so the above implementation is a completely ideal implementation and both the performance requirement and the algorithm complexity are controllable.

The signal processing algorithm flow of LTE simulator is as shown in Figure 1, and the algorithm framework is designed according to literature [11]. Differently, the dotted modules only belong to the improved algorithm in this paper, including non LTE standard module, frequency reduction module, modified frame generator module and channel estimation module configured for two-dimensional spread spectrum. The full-line modules are the modules owned by existing algorithms, as shown in Figure 2. Please refer to literature [11] for the English abbreviations not repeated in this section.

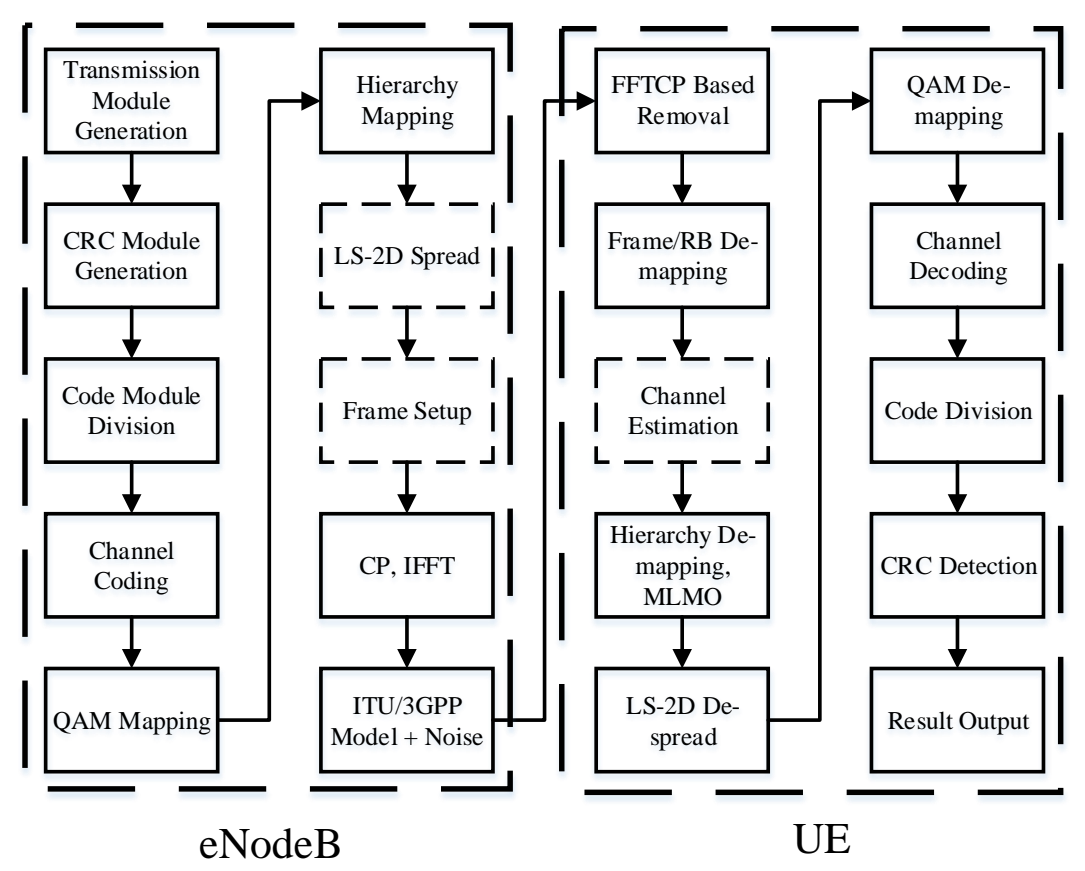

Figure 2. LTE signal Processing Chain

$n$-dimensional receiving signal vector $\mathbf{y} \in \mathrm{C}^{\mathrm{l} \times n}$ is defined as follows:

$$
\mathbf{y}=\mathbf{Z h}+\mathbf{v}
$$

Where $\mathbf{h} \in \mathrm{C}^{1 \times n}$ is the channel parameter vector, $n$ is the quantity of the transmission code elements and is related to bandwidth BW. As the additional white Gaussian noise, $\mathbf{v} \in \mathrm{C}^{1 \times n}$ is set as $\mathbf{v}=\left[v_{1}, v_{2}, \cdots, v_{n}\right]$ and the elements thereof can be defined as follows:

$$
v_{i}=\sqrt{N_{f f t} / N_{t o t}} 10^{-\frac{S N R}{20}}(N(0,1)+j N(0,1))
$$

Where $N(0,1)$ refers to the normal random number distribution with the mean value as 0 and the variance as $1, j$ is the imaginary number unit, Crepresents the complex field, and $\sqrt{N_{f f t} / N_{\text {tot }}}$ is adopted for LTE to compensate for the so called zero subcarrier. 


\section{Linear LS-2D Spread Spectrum Model}

\subsection{Algorithm Model}

As mentioned above, according to the two-dimensional spread spectrum algorithm characteristics and the time-frequency domain LTE resource model mentioned in Chapter I, data fusion is implemented for multiple LTE resource models through adding the weight factor and meanwhile the linear LS algorithm is adopted to normalize the weight factor and 2D spread spectrum power, as shown in Figure 3.

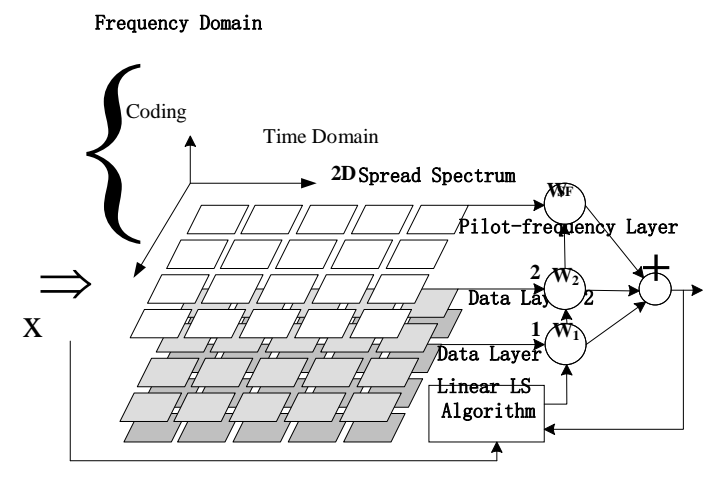

Figure 3. Linear LS-2D Spread Spectrum Channel Estimation Model

In the figure, $w_{i}$ is the element of vector $w \in R_{>0}^{1 \times S F}$ and is used to adjust the weight factor of SNR (Signal to Noise Ratio) of each layer. Here, the new channel estimation algorithm is adopted to simplify the frame generator module, so the spread spectrum data symbols are arranged in a stack form. Since the data code elements are spread according to the spread spectrum factor SF, and the quantity of the transmission chips (spread spectrum data symbols) is $S F$ times of the quantity of the code elements and is set as $e S F$. At the receiving terminal, due to the orthogonality of Hadamard sequence, the code layer can be separated. Since the spread spectrum signals have addition operation in the code domain, thus the signal with length as $n$ can be obtained. The length of the obtained signal is equal to that of the signal under standard LTE model, so the bandwidth of the spread spectrum signal is also equal to that of the standard LTE link.

As shown in Figure 3, the first $S F-1$ code layers are used for data transmission and the $S F^{\text {th }}$ layer is the pilot-frequency layer, and the data are expressed as $\mathbf{x}_{\mathbf{p}}$.

\subsection{Linear LS Algorithm}

In Formula (1), parameter $\mathbf{Z}$ is a diagonal matrix and can be defined as follows:

$$
\mathbf{Z}=\left[\begin{array}{ccc}
z_{1} & 0 & 0 \\
0 & \ddots & 0 \\
0 & 0 & z_{n}
\end{array}\right]
$$

Where $\mathbf{z}=\left[z_{1}, \cdots, z_{n}\right]$ is the complex vector of the permutation data and the pilot frequency and can be defined as follows:

$$
\mathbf{z}=\mathbf{P} \mathscr{Z}_{o}=\mathbf{P}\left[\mathbf{z}_{p}^{T}, \mathbf{z}_{d}^{T}\right]^{T}
$$

Where $\mathbf{P} \in N_{0 \leq 1}^{n \times n}$ is a standard permutation matrix. LS estimation can be defined as follows:

$$
\hat{\mathbf{h}}_{p}^{L S}=\mathbf{Z}_{p}^{H} \mathbf{y}_{p}
$$


Where $\mathbf{Z}_{p}^{H}$ is Hermitian transposition of the transmitted signal matrix $\mathbf{Z}$ at the specific pilot-frequency position, $\mathbf{y}_{p} \in \mathrm{C}^{\mathrm{l} \times p}$ is the received pilot-frequency signal with the length as $p$. The channel coefficient of the pilot-frequency code elements can be obtained through interpolation.

In order to simplify computer implementation and improve the algorithm operation efficiency, the simple linear LS algorithm is adopted for the implementation. According to the description in literature [12], LS filter can be defined as follows:

$$
\left\{\begin{array}{l}
\hat{\mathbf{h}}_{L S}=\mathbf{A}_{L S} \hat{\mathbf{h}}_{p}^{L S} \\
\mathbf{A}_{L S}=\mathbf{R}_{h, h_{p}}\left(\mathbf{R}_{h_{p}, h_{p}}+\sigma^{2} \mathbf{I}\right)^{-1}
\end{array}\right.
$$

Where $\mathbf{I}$ is the unit matrix, $\sigma^{2}$ is the noise variance, $\mathbf{R}_{h, h_{p}}$ is the channel crosscovariance matrix and $\mathbf{R}_{h_{p}, h_{p}}$ is the channel covariance matrix. Under ideal conditions, these variables are assumed to be known or can be estimated through the training sequences.

\subsection{Linear LS-2D Spread Spectrum}

This section mainly aims at describing LTE system model based on the improved 2D spread spectrum. The receiving signal $\mathbf{y} \in \mathrm{C}^{\mathrm{1} \times n}$ can be defined as follows:

$$
\mathbf{y}=\left(y_{1}, y_{2}, \cdots, y_{n}\right)
$$

Similarly to the traditional CDMA receiver, the signal vector can be obtained through the de-spread matrix $Q \in N_{0 \leq 1}^{S F \times S F}$. Firstly, Hadamard matrix is multiplied with the spread spectrum matrix:

$$
\left\{\begin{array}{l}
\mathbf{Y}=\left(\mathbf{y}_{1}, \mathbf{y}_{2}, \cdots, \mathbf{y}_{S F}\right)^{T} \\
\mathbf{y}_{i}=\left(\mathbf{y} \square Q_{(i, *)}\right) / S F
\end{array}\right.
$$

Where $\square$ represents Hadamard product, the de-spread matrix $Q$ is $n / S F$-order copy matrix and can be defined as follows:

$$
Q=\left[\theta_{1}, \theta_{2}, \cdots, \theta_{n / S F}\right]
$$

Output vector $\mathbf{Y}_{\mathrm{d}} \in \mathrm{C}^{n}$ can be composed of some elements of data matrix $\mathbf{Y} \in \mathrm{C}^{\mathrm{n} \times S F}$ :

$$
\left\{\begin{aligned}
\mathbf{Y}_{\mathbf{d}}= & \left(\mathbf{y}_{d 1}, \mathbf{y}_{d 2}, \cdots, \mathbf{y}_{d n / S F}\right)^{T} \\
& \left(\begin{array}{l}
\sum_{k=1}^{S F}(y(k, S F(i-1))+1), \\
\mathbf{y}_{d i}= \\
\sum_{k=1}^{S F}(y(k, S F(i-1))+2), \\
\cdots, \sum_{k=1}^{S F} y(k, i \cdot S F)
\end{array}\right)
\end{aligned}\right.
$$

In consideration of the unit pilot-frequency vector, the channel estimation can be expressed as the $S F^{\text {th }}$ code layer of matrix $\mathbf{Y}_{\mathbf{d}}$. Since the pilot-frequency layer is transmitted in a spread spectrum form, in order to realize the complete CSI estimation in the time-frequency resources, it is necessary to spread the pilotfrequency layer again. Therefore, the pilot-frequency layer shall not be interpolated and can be defined as follows:

$$
\mathbf{h}^{2 D}=\mathbf{y}_{d n / S F} \otimes Q_{(S F, *)}
$$

Vector $\mathbf{x} \in \mathrm{C}^{\mathrm{d} \times n}$ of the signal from the hierarchy mapping module, as shown in Figure 3, can be defined as follows:

$$
\mathbf{x}=\left[\mathbf{x}_{d}^{T}, \mathbf{x}_{p}^{T}\right]^{T}
$$


Where $\mathbf{x}_{d}^{T} \in \mathrm{C}^{\mathrm{I} \times(n-p)}$ is the data code element vector, $\mathbf{x}_{p}^{T} \in \mathrm{C}^{\mathrm{1} \times p}$ is the pilot-frequency code element vector, $n$ is corresponding to the system bandwidth and $p$ is the quantity of the subcarriers. Received signal $\mathbf{y}$ is defined similarly to Formula (1). As the diagonal matrix for describing the pilot-frequency and the data for spread spectrum operations, $\mathbf{X}_{s} \in \mathrm{C}^{n \times n}$ can be defined as follows:

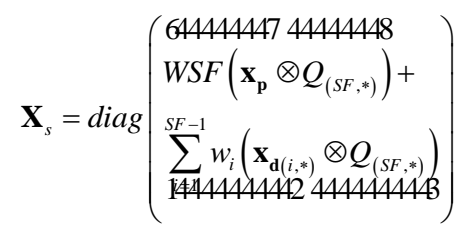

Where relevant parameter vector is calculated as follows:

$$
\left\{\begin{array}{l}
\mathbf{x}_{\mathrm{d}}=\left[\begin{array}{ccc}
x_{1} & \cdots & x_{n} / S F \\
\vdots & \ddots & \vdots \\
x_{n} / S F+1 & \cdots & x_{n}
\end{array}\right] \\
Q=\left[\begin{array}{ccc}
\varsigma_{1,1} & \cdots & \varsigma_{1, S F} \\
\vdots & \ddots & \vdots \\
\varsigma_{S F, 1} & \cdots & \varsigma_{S F, S F}
\end{array}\right]
\end{array}\right.
$$

Where $S F$ is the two-dimensional spread spectrum factor, $Q \in N_{0 \leq 1}^{S F S F}$ is SF-order Hadamard matrix, $\otimes$ is Kronecker product, $\mathbf{x}_{\mathbf{d}(i, *)}$ represents the $i$ th line of matrix $\mathbf{x}_{\mathrm{d}}$ which is the pilot-frequency sequence set as $\mathbf{x}_{\mathbf{p}}=\mathbf{1}_{1, n / S F}$ for simplification, namely the unit vector with the length as $n / S F$.

Since there is no need to obtain any prior information, the calculation complexity of the proposed algorithm is basically similar to that of LS method and the twodimensional spread spectrum algorithm.

Table 1. LTE System Parameter Setting

\begin{tabular}{cc}
\hline Parameter & Set Value \\
\hline Number of Code Elements in Each Frame & $n \in(826,982)$ \\
Quantity of Sub-frames (Duration) & $1000(1 \mathrm{~ms})$ \\
$N_{m} / N_{t u}$ & $128 / 72$ \\
SF & 48 \\
System Bandwidth BW & $1.3 \mathrm{MHz}$ \\
User End (UE) Rate & $15 \mathrm{kM} / \mathrm{h}$ \\
UE Quantity & 1 \\
Carrier Frequency & $2.3 \mathrm{GHz}$ \\
Subcarrier Interval & $15 \mathrm{kHz}$ \\
eNodeBs Quantity & 1 \\
\hline
\end{tabular}

\section{Simulation Experiment and Analysis}

The comparison simulation is implemented in Wiener LTE down-link simulator and the simulation parameters are set as shown in Table 1. The channel models for comparison include: VehA, VehB, ETU, RA, PedA, PedB, HT, etc.; please refer to literature [13] for the details. The hardware simulation platform is as follows: processor: i7-4790K 4GHz; memory: Kingston 8G ddr3 1600; operating system: Windows7; simulation software: matlab2013b. The simulation experiment is carried out on the basis of the following estimations:

(1) Covariance matrix based linear least square algorithm (CMILLS); 
(2) Covariance matrix training sequence based linear minimum mean square error estimation (CMSLLS);

(3) Least square (LS) estimation;

(4) Two-dimensional spread spectrum (2D spread spectrum) estimation method.

In the above algorithms, the root mean square error (MSE) of the estimated value and the actual value can be regarded as the index for evaluating the algorithm estimation accuracy. Additionally, LTE-OFDM multi-layer channel estimation algorithm based on the linear least square 2D spread spectrum proposed in this paper is also implemented in the experiment. Specifically, SNR value of the algorithms mentioned above is selected as X-axis, wherein CMSLLS is an estimation algorithm for ideal conditions and MSE value thereof is regarded as the comparison simulation target. The simulation comparison results are as shown in Figure 4.

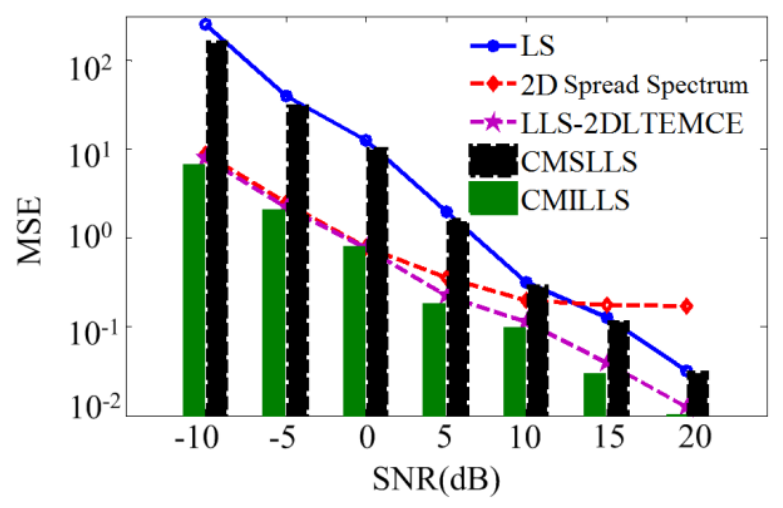

Figure 4. Comparison of MSE based SNR change

According to Figure 4, among all comparison algorithms, CMILLS algorithm has the minimum MSE value and the best performance, but this algorithm has a defect that the second-order statistical knowledge of the wireless channel, namely the channel covariance information, shall be completely known, but it is difficult to meet such condition in practical operation, and this algorithm is especially sensitive to the interference. In this paper, MSE value calculated under ideal conditions is regarded as the basis for evaluating the performances of other algorithms.

According to the analysis of the simulation results shown in Figure 4, the least square estimation algorithm has the poorest accuracy among all comparison algorithms, and the covariance matrix training sequence based linear least square mean square error estimation has similar accuracy and relatively low performance. Under high SNR, MSE values of the two-dimensional spread spectrum estimation algorithm and the proposed algorithm are very approximate to MSE convergence value under ideal conditions of CMILLS; but along with the reduction of SNR, the two-dimensional spread spectrum gradually becomes saturated, and MSE value of LLS-2DLTEMCE algorithm is always approximate to MSE convergence value under the ideal conditions of CMILLS, thus proving the advantages of LLS2DLTEMCE algorithm in the aspect of the robustness and the convergence accuracy.

The selected channel models are as follows: ETU, RA and PedA. SNR based network throughput changes of the above algorithms are as shown in Figure 4. According to the algorithm performance comparison, LS and CMSLLS algorithms have poor estimation accuracy. Here, only CMSLLS algorithm is selected for the network throughput comparison, but CMILLS algorithm is the algorithm designed 
for ideal conditions, so it is not selected. Specifically, the comparison results are as shown in Figures 5 (a) (c).

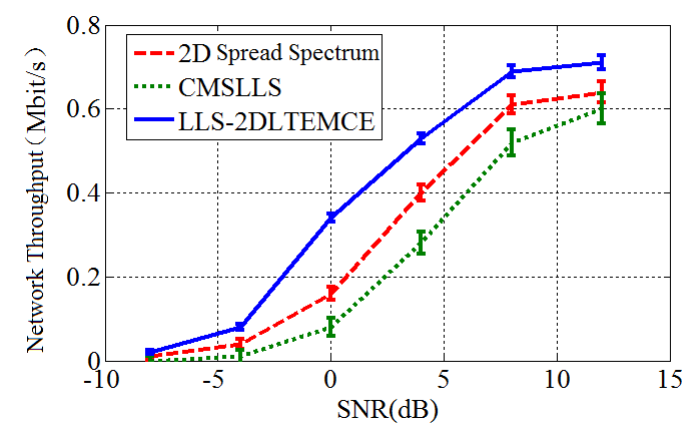

(a) Network throughput Comparison of ETU Channel Models

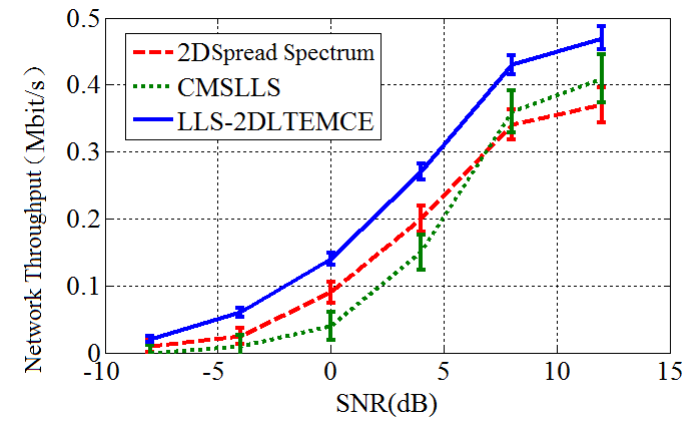

(b) Network throughput Comparison of RA Channel Models

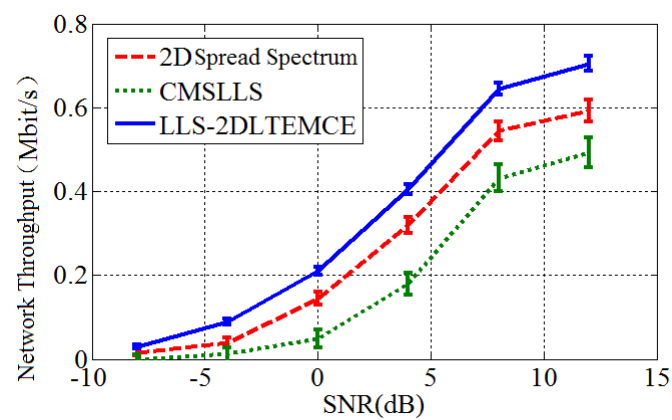

(c) Network throughput Comparison of Peda Channel Models

Figure 5. Network Throughput Simulation Comparison

The network throughput can reflect the network data processing capacity, and the network throughput comparison results and the change curves of CMSLLS, 2D spread spectrum and LLS-2DLTEMCE algorithms in ETU, RA and PedA models are as shown in Figures 5 (a) (c). According to Figures (a) (c), the proposed LLS-2DLTEMCE algorithm is always superior to CMSLLS and 2D spread spectrum algorithms in the aspect of the network throughput. As shown in Figure (a), along with the increase of SNR value, the network throughput advantage of LLS-2DLTEMCE algorithm gradually becomes obvious from $S N R=-10 \sim-5$ stage to $S N R=-5 \sim 5$ stage and then gradually becomes weak since $S N R=5 \sim 15$ stage; generally speaking, LLS-2DLTEMCE algorithm is always superior to other two algorithms. As shown in Figure (c), the overall tendency is similar to that in Figure (a), the algorithms do not have obvious advantages in $S N R=-10 \sim-5$ stage, but LLS2DLTEMCE algorithm gradually has obvious advantage in $S N R=-5 \sim 15$ stage. As shown in Figure (b), 2D spread spectrum algorithm is superior to CMSLLS algorithm in the aspect of the network throughput in early stage $(S N R=-10 \sim 7)$ but 
inferior to CMSLLS algorithm in the same aspect in later stage ( $S N R=7 \sim 15$ ); generally speaking, LLS-2DLTEMCE algorithm is always superior to the above two algorithms. The simulation result indicates that the improvement of the convergence accuracy (MSE) of LLS-2DLTEMCE algorithm is not realized at the cost of sacrificing the network throughput, and this presents the overall improvement of the algorithm performance.

During the signal transmission process, especially during terminal movement, the signal strength is increased for the coming terminal but reduced for the leaving terminal. Such phenomenon exists in various fields and is called as "Doppler Effect". Doppler effect simulation comparison result of the network throughput along with the terminal movement is as shown in Figure 6, wherein the simulation parameters are as follows: $S N R=2$; terminal movement speed $v=0 \sim 400 \mathrm{kM} / \mathrm{h}$; other parameters are selected as shown in Table 1. CMSLLS and 2D spread spectrum algorithms are also selected as the comparison algorithms. The simulation result is as shown in Figure 6.

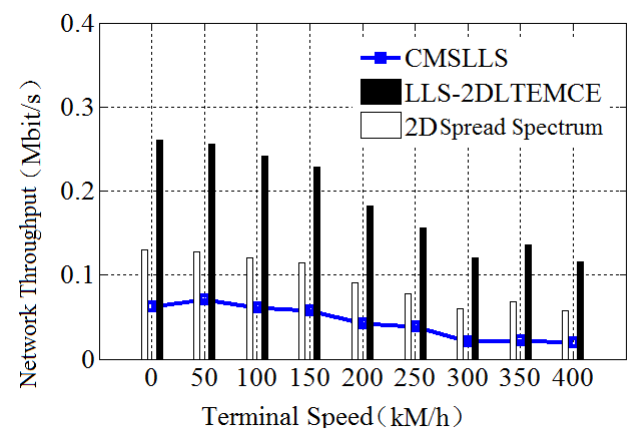

Figure 6. Network Throughput Doppler Effect

Doppler effect simulation comparison results of the three algorithms are as shown in Figure 6. According to the figure, along with the increase of the terminal movement speed, the network throughputs of the three algorithms are all influenced and generally present a reduction tendency. At some moments, for example, at $v=300 \mathrm{kM} / \mathrm{h}$, the network throughput is sharply reduced and then increased more or less; but, generally speaking, LLS-2DLTEMCE algorithm is superior to the comparison algorithms in the aspect of the network throughput in the whole terminal movement speed range, namely within $v=0 \sim 400 \mathrm{kM} / \mathrm{h}$.

\section{Conclusion}

In allusion to the two indexes ---- LTE channel estimation accuracy and network throughput, this paper mainly aims at designing the algorithm improvement mode. Specifically, the weighted multi-layer time-frequency domain LTE-OFDM resource model is adopted to design the linear least square algorithm for weight parameter estimation and 2D spread spectrum normalization algorithm. The result of the simulation comparison in the standard model database shows that the proposed algorithm can not only improve the algorithm estimation accuracy, but also keep high network throughput, thus verifying the algorithm effectiveness. After the algorithm feasibility is experimentally verified, it is necessary to research the establishment of the hardware platform and the operation effect of the algorithm in such platform so as to verify the practical application effect of the algorithm. 


\section{References}

[1] J. Hu and Z. Gao, "Modules identification in gene positive networks of hepatocellular carcinoma using Pearson agglomerative method and Pearson cohesion coupling modularity", Journal of Applied Mathematics, (2012).

[2] Z. Lv, A. Tek and F. D. Silva, "Game on, science-how video game technology may help biologists tackle visualization challenges", PloS one, vol. 8, no. 3, (2013), p. 57990.

[3] T. Su, W. Wang and Z. Lv, "Rapid Delaunay triangulation for randomly distributed point cloud data using adaptive Hilbert curve", Computers \& Graphics, vol. 54, (2016), pp. 65-74.

[4] J. Hu, Z. Gao and W. Pan, "Multiangle Social Network Recommendation Algorithms and Similarity Network Evaluation", Journal of Applied Mathematics, (2013).

[5] S. Zhou, L. Mi, H. Chen and Y. Geng, "Building detection in Digital surface model", 2013 IEEE International Conference on Imaging Systems and Techniques (IST), (2012).

[6] J. He, Y. Geng and K. Pahlavan, "Toward Accurate Human Tracking: Modeling Time-of-Arrival for Wireless Wearable Sensors in Multipath Environment”, IEEE Sensor Journal, vol. 14, no. 11, (2014), pp. 3996-4006.

[7] Z. Lv, A. Halawani and S. Fen, "Touch-less Interactive Augmented Reality Game on Vision Based Wearable Device", Personal and Ubiquitous Computing, vol. 19, no. 3, (2015), pp. 551-567.

[8] G. Bao, L. Mi, Y. Geng, M. Zhou and K. Pahlavan, "A video-based speed estimation technique for localizing the wireless capsule endoscope inside gastrointestinal tract", 2014 36th Annual International Conference of the IEEE Engineering in Medicine and Biology Society (EMBC), (2014).

[9] D. Zeng and Y. Geng, "Content distribution mechanism in mobile P2P network", Journal of Networks, vol. 9, no. 5, (2014), pp. 1229-1236.

[10] W. Gu, Z. Lv and M. Hao, "Change detection method for remote sensing images based on an improved Markov random field", Multimedia Tools and Applications, (2015), pp. 1-16.

[11] Z. Chen, W. Huang and Z. Lv, "Towards a face recognition method based on uncorrelated discriminant sparse preserving projection", Multimedia Tools and Applications, (2015), pp. 1-15.

[12] J. $\mathrm{Hu}$ and Z. Gao, "Distinction immune genes of hepatitis-induced hepatocellular carcinoma", Bioinformatics, vol. 28, no. 24, (2012), pp. 3191-3194.

[13] T. Su, W. Wang and Z. Lv, "Rapid Delaunay triangulation for randomly distributed point cloud data using adaptive Hilbert curve", Computers \& Graphics, vol. 54, (2016), pp. 65-74.

[14] W. Gu, Z. Lv and M. Hao, "Change detection method for remote sensing images based on an improved Markov random field", Multimedia Tools and Applications, (2015), pp. 1-16.

[15] Z. Lv, A. Tek and F. D. Silva, "Game on, science-how video game technology may help biologists tackle visualization challenges", PloS one, vol. 8, no. 3, (2013), p. 57990.

[16] Z. Chen, W. Huang and Z. Lv, "Towards a face recognition method based on uncorrelated discriminant sparse preserving projection", Multimedia Tools and Applications, (2015), pp. 1-15.

[17] D. Jiang, X. Ying and Y. Han, "Collaborative multi-hop routing in cognitive wireless networks", Wireless Personal Communications, (2015), pp. 1-23.

[18] Z. Lv, A. Tek and F. D. Silva, "Game on, science-how video game technology may help biologists tackle visualization challenges", PloS one, vol. 8, no. 3, (2013), p. 57990.

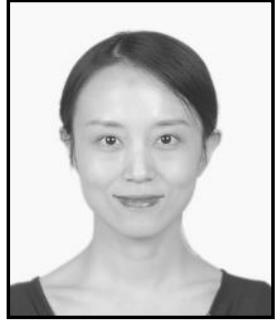

\section{Authors}

Li Wenjuan, she received her M.S. degree in Communication and Information System from Liaoning Technical University in Liaoning, China. She is currently a lecturer in the College of Mobile Telecommunications Chongqing University of Posts and Telecom. Her research interest is mainly in the area of Mobile Communication, Signals and Systems. She has published several research papers in scholarly journals in the above research areas and has participated in several books. 